\title{
Nematic Liquid Crystal Waveguides for Spatial Control of Linearly Polarized Light Waves
}

\author{
V. S. Kabanava, I. I. Rushnova, E. A. Melnikova, and A. L. Tolstik \\ Belarusian State University, 4 Niezaliežnsci ave., Minsk, Belarus
}

\begin{abstract}
Electrically switchable liquid crystal (LC) waveguide structures for spatial-polarization control of light waves become more and more widespread as optical components for waveguide photonics. The motivation of the work was to design and fabricate miniaturized, low-cost electrically switchable LC waveguides for spatial control of linearly polarized light beams. With the use of new configurations of the spatially modulated electric field applied to planar-aligned sandwich-type LC cells $(d=20 \mu \mathrm{m}$ thick $)$, the polarization-sensitive waveguide structures performing the functions of optical splitter and adder were designed and experimentally studied. The current-conducting glass substrates coated with patterned electrodes (splitter-shaped and adder-shaped chromium layers) were used to control the optical properties of LC waveguides by applying an external low-frequency $(1 \mathrm{kHz})$ electrical voltage to the LC cell electrodes. The operation principle of the fabricated optical components is based on the effect of total internal reflection (TIR) of laser radiation from the electrically controlled refractive interface between LC areas with orthogonal director orientations. Optical components of this type look promising for the production of competitive low power consumption photonic devices with enhanced functional characteristics.
\end{abstract}

\section{INTRODUCTION}

Liquid crystal (LC) materials have found wide application in the field of designing and manufacturing optical devices whose operation principle is based on the manifestation of linear and nonlinear optical effects. In the last decades, in order to solve both the research and applied problems of integrated optics, great attention has been devoted to the LC waveguide structures for routing of light waves in the visible and near infrared spectrum ranges [1-4]. Current topics in the field of optical LC waveguides cover a wide range of research problems related to: choice of optimal LC-materials; development of LC alignment technologies; design of new LC waveguide geometries; innovative methods to design and manufacture the LC waveguide optical components characterized by enhanced functional characteristics; simplification of the process of LC components integration into integrated optical circuits.

This work deals with electrically switchable nematic LC waveguide structures providing controlled functions of transmission, splitting and multiplexing of linearly polarized light beams in the visible spectral range.

\section{ELECTRICALLY SWITCHABLE LC WAVEGUIDES BASED ON PATTERNED ELECTRODE}

The periodic waveguide refractive index distribution within LC media can be realized by applying an external spatially modulated electric field to the thin planar-aligned LC layer [5-10]. In order to generate a spatially modulated electric field, the patterned current-conductive layers (patterned electrodes) deposited on the LC cell substrate are usually used. Shape of the patterned electrode, in turn, determines the type of spatial modulation for the electric field strength applied to the LC cell.

Figure 1 shows a schematic diagram of the electrically switchable LC cell with a patterned (combshaped) electrode on the bottom glass substrate. A glass plate uniformly coated with a transparent conductive layer of indium tin oxide (ITO) was used as the upper cell substrate. The LC layer thickness was set at $d=20 \mu \mathrm{m}$. The initial planar alignment of the LC director along $z$-axis was realized by photostimulated rubbing of photosensitive benzaldehyde polymer films [11]. Filling of the cell with a nematic LC material $\left(n_{o}=1.49, n_{e}=1.67\right.$ at $\left.\lambda=632.8 \mathrm{~nm}\right)$ was performed under isotropic phase condition $\left(T=75^{\circ}\right)$. In order to induce the periodic refractive index distribution within a planar-aligned nematic LC layer, the electric field spatially modulated along the $y$-axis was applied to the cell electrodes.

We will consider the propagation of linearly polarized laser radiation $(\mathbf{E}\|x, \mathbf{k}\| z)$ in the plane of a thin-film nematic LC layer at different applied voltages $U$. In voltage-off-state $(U=0)$ the 


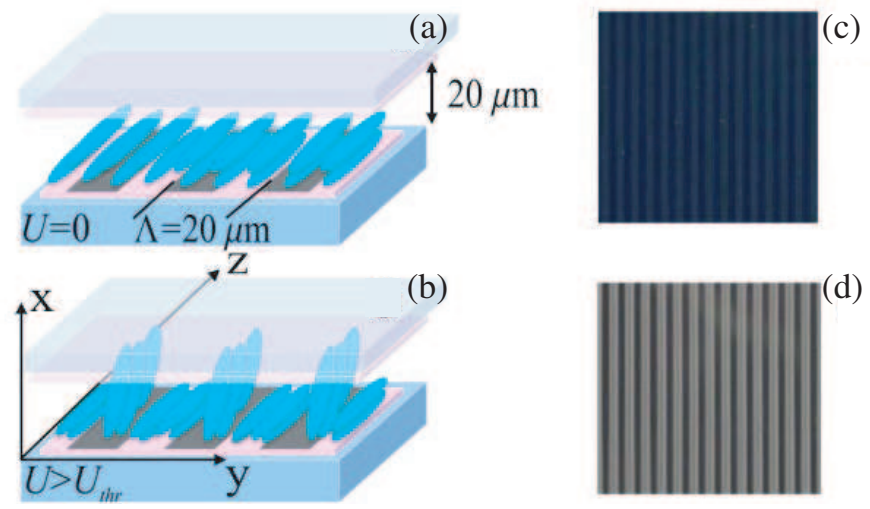

Figure 1: Electrically switchable LC cell with patterned (comb-shaped) electrode: (a), (b) schematic diagram and (c), (d) corresponding polarized microphotographs.

LC director $(\mathbf{n})$ is planar aligned along $z$-axis $(\mathbf{n} \| z)$, as shown in Fig. 1, therefore, the refraction index of nematic LC corresponds to $n_{o}=1.49$ since $\mathbf{n} \perp \mathbf{E}$. At voltages above the threshold value $\left(U>U_{t h r}\right)$ in the region of conductive periodic strips the Fredericks transition is realized leading to the LC director reorientation from planar to homeotropic position. (i.e., the pretilt angle $\theta$ increases). As the pretilt angle $(\theta)$ is increasing, the LC effective refraction index $\left(n_{e f}\right)$ in the core region of the $\mathrm{LC}$ waveguide increases too according to the equation:

$$
n_{e f}(\theta)=\frac{n_{e} \cdot n_{o}}{\sqrt{n_{e}^{2} \cdot \cos ^{2} \theta+n_{o}^{2} \cdot \sin ^{2} \theta}} .
$$

At the same time, in the area between the conductive strips the refraction index of LC medium for laser radiation linearly polarized along the $x$-axis corresponds to $n_{o}=1.49$, because the LC director orientation remains unperturbed (i.e., planar). The formation of electrically induced refractive interfaces between LC areas with different director orientations makes it possible to implement the waveguide mode propagation of light by means of TIR effect within the LC core region.

New configurations of patterned electrodes (Fig. 2) have been proposed in order to fabricate miniaturized electrically-switchable LC waveguides operating as optical splitters and adders.

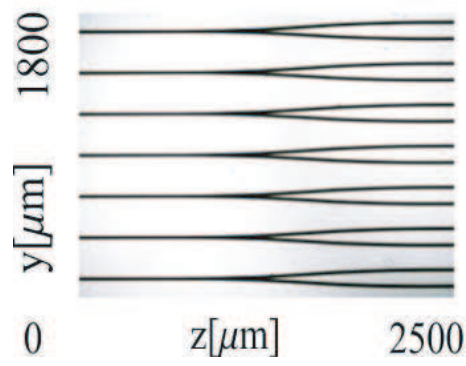

(a)

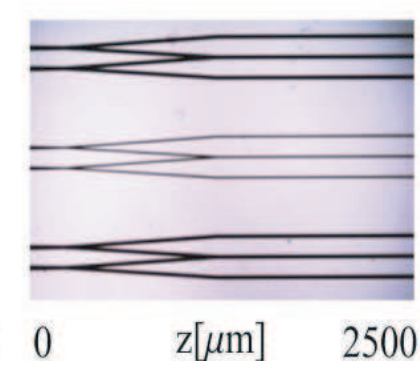

(b)

Figure 2: Microphotographs of glass substrates covered with (a) splitter-shaped and (b) adder-shaped patterned electrodes.

\section{EXPERIMENTAL RESULTS}

Figure 3(a) shows experimental microphotographs of a laser beam propagation $(\lambda=532 \mathrm{~nm}$, $300 \mu \mathrm{W}$ power, $\mathbf{E}\|x, \mathbf{k}\| z)$ in the LC cell with a splitter-shaped patterned electrode at different applied voltages. The corresponding light beam intensity distribution profiles $I(y)$ for the propagation length $z=2.0 \mathrm{~mm}$ are shown in Fig. 3(b). In voltage-off-state $(U=0)$, laser radiation is scattered within a planar-aligned LC layer - the cell operates in the light beam attenuation mode. In this case, the refractive index of LC media corresponds to $n_{o}=1.49$. When an external voltage exceeds the threshold value $(U>1.0 \mathrm{~V})$, one can observe the formation of the waveguide LC channels the geometry of which is corresponds to the geometry of a patterned electrode. The 


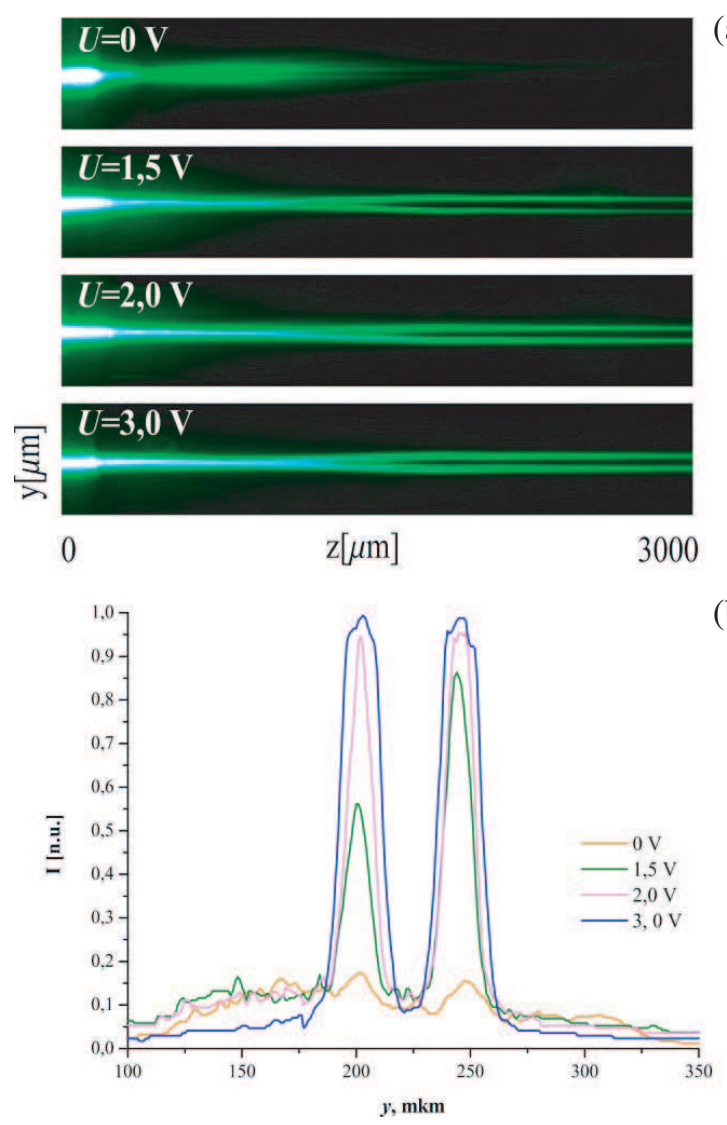

(a)

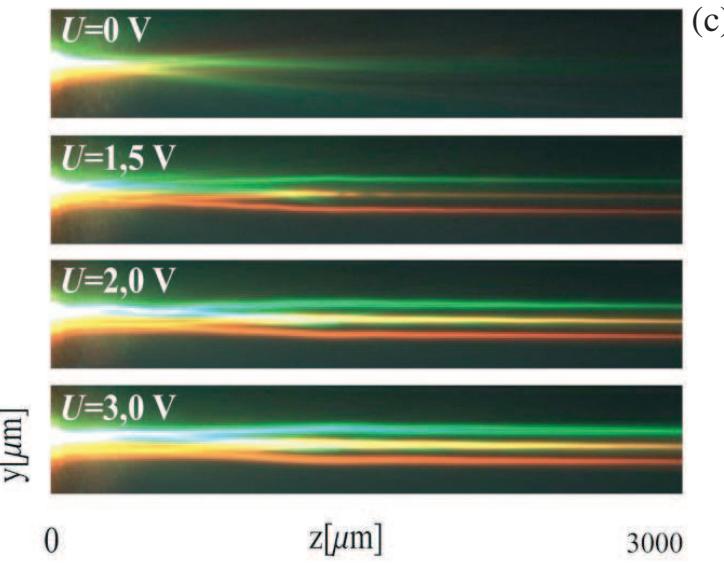

(b)

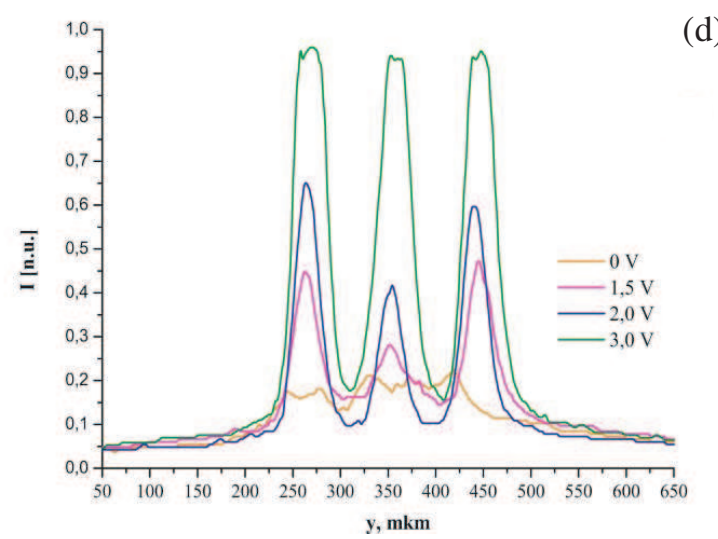

Figure 3: Spatial control of linearly polarized light beams by means of electrically switchable nematic LC waveguides: (a), (b) light beam propagation in waveguide LC splitter (top-view microphotographs)and corresponding light intensity distribution profiles $I(y)$ at $z=2.0 \mathrm{~mm}$ for different applied voltages $U$; (c), (d) cooperative light beam propagation in the waveguide LC adder (top view microphotographs) and corresponding light intensity distribution profiles $I(y)$ at $z=2.0 \mathrm{~mm}$ for different applied voltages $U$.

LC director reorientation (from planar to homeotropic position) under the influence of an external spatially modulated electric field causes an increase of the effective refraction index in the core region of a LC waveguide splitter. The electrically switchable LC waveguide splitter enables one to implement the spatial control of laser beams as well as to realize the functions of the opticalpower divider. While the waveguide mode is implemented only for light beams with polarization corresponding to an extraordinary wave, a waveguide LC splitter may be also used as an integratedoptical polarizer as well.

Figure 3(c) shows experimental microphotographs of the cooperative laser beams propagation $\left(\lambda_{1}=532 \mathrm{~nm}, \lambda_{2}=633 \mathrm{~nm}, 300 \mu \mathrm{W}\right.$ powers, $\left.\mathbf{E}_{1,2}\left\|x, \mathbf{k}_{1,2}\right\| z\right)$ in an LC cell with the adder-shaped patterned electrode for different applied voltages. The corresponding light beam intensity distribution profiles $I(y)$ for the propagation length $z=2.0 \mathrm{~mm}$ are shown in Fig. 3(d). In the switched-off state $(U=0)$ the cell is operating as an optical attenuator. The use of the adder shaped patterned electrode offers separation of the input light beams into two identical optical channels when the applied voltage exceeds the threshold value $(U>1,5 \mathrm{~V})$. When light beams propagate in the waveguide mode within the plane of an LC layer at the central region of the patterned electrode, their optical powers are added. As a result, an electrically induced waveguide LC adder provides the externally controlled optical power separation/multiplication functions as well as the integrated optical polarizer function characterized by low operating voltages $(1.5-4.0 \mathrm{~V})$.

\section{CONCLUSION}

This paper presents a relatively simple technique to design electrically switchable LC waveguide structures for spatial control of linearly polarized light waves. It has been experimentally demonstrated that designed LC waveguides provide the externally controlled optical power splitting/multiplexing functions and the integrated optical polarizer function characterized by low operating 
voltages $(1.5-4.0 \mathrm{~V})$. The obtained results are promising for design and fabrication of competitive, reliable photonic devices aimed at spatial-polarization routing of light waves.

\section{ACKNOWLEDGMENT}

The research was supported by the Belarusian State Program of Scientific Investigations "Convergence 2016-2020" (task 3.03.5).

\section{REFERENCES}

1. Zografopoulos, D. C., R. Asquini, E. E. Kriezis, A. d'Alessandro, and R. Beccherelli, "Guidedwave liquid-crystal photonics," Lab Chip, Vol. 12, 3598-3610, 2012.

2. Rutkowska, K. A., M. Chychłowski, M. Kwasny, I. Ostromęcka, J. Piłka, and U. Laudyn, "Light propagation in periodic photonic structures formed by photo-orientation and photopolymerization of nematic liquid crystals," Opto-Electronics Review, Vol. 25, No. 2, 118-126, 2017.

3. Asquini, R., D. Donisi, M. Trotta, A. d'Alessandro, B. Bellini, G. Gilardi, and R. Beccherelli, "Realization of a liquid crystal electrically controlled optical waveguide on micromachined silicon," Mol. Cryst. Liq. Cryst., Vol. 500, 23-30, 2009.

4. Wang, T.-J., C.-K. Chaung, W.-J. Li, T.-J. Chen, and B.-Y. Chen, "Electrically tunable liquid-crystal-core optical channel waveguide," J. Light. Technol., Vol. 31, 357-3574, 2013.

5. Bouvier, M. and T. Scharf, "Analysis of nematic-liquid-crystal binary gratings with high spatial frequency," Opt. Eng., Vol. 39, 2129-2137, 2000.

6. Brzdąkiewicz, K. A., M. A. Karpierz, A. Fratalocchi, G. Assanto, and E. NowinowskiKruszelnicki, "Nematic liquid crystal waveguide arrays," Opto-Electronics Rev., Vol. 13, No. 2, 107-112, 2005.

7. Maksimochkin, A. G., S. V. Pasechnik, G. I. Maksimochkin, and V. G. Chigrinov, "Electrically controlled waveguide mode in LC layer for fiber optic applications," Opt. Commun., Vol. 283, No. 16, 3136-3141, 2010.

8. Wang, T.-J., C.-K. Chaung, W.-J. Li, T.-J. Chen, and B.-Y. Chen, "Electrically tunable liquidcrystal-core optical channel waveguide," J. Lightwave Technol., Vol 31, 3570-3574, 2013.

9. Shenoy, M. R., M. Sharma, and A. Sinha, "An electrically controlled nematic liquid crystal core waveguide with a low switching threshold," J. Lightwave Technol., Vol. 33, 1948-1953, 2015.

10. Assanto, G., A. Fratalocchi, and M. Peccianti, "Spatial solitons in nematic liquid crystals: From bulk to discrete," Opt. Express, Vol. 15, No. 8, 5248-5259, 2007.

11. Mahilny, U. V., A. I. Stankevich, A. V. Trofimova, A. A. Muravsky, and A. A. Murauski, "Photosensitive polymers for liquid crystal alignment," Phys. Procedia, Vol. 73, 121-125, 2015. 